\title{
FOURIER INVERSION FOR SEMISIMPLE LIE GROUPS OF REAL RANK ONE ${ }^{1}$
}

\author{
BY P. J. SALLY, JR. AND GARTH WARNER \\ Communicated by Ray A. Kunze, August 4, 1971
}

1. Introduction. Let $G$ be a connected semisimple Lie group with finite center and let $K$ be a maximal compact subgroup of $G$. We assume that $\operatorname{rank}(G)=\operatorname{rank}(K)$ and that $\operatorname{rank}(G / K)=1$. Let $T$ be a Cartan subgroup of $G$ contained in $K$ and let $A$ be the noncompact Cartan subgroup of $G$ constructed in [3f), §24]. Then $(T, A)$ is a complete set of nonconjugate Cartan subgroups of $G$. We set $T^{\prime}=T \cap G^{\prime}, A^{\prime}=A \cap G^{\prime}$, where $G^{\prime}$ denotes the set of regular elements in $G$. We write $g$ and $t$ for the Lie algebras of $G$ and $T$, and $\mathfrak{g}_{\boldsymbol{c}}, \mathrm{t}_{\boldsymbol{c}}$ for the complexifications of $\mathfrak{g}$ and t. If $G_{\boldsymbol{C}}$ is the simply connected complex analytic group corresponding to $\mathfrak{g}_{c}$, we assume that $G$ is the real analytic subgroup of $G_{\boldsymbol{C}}$ corresponding to $\mathfrak{g}$.

For $f \in C_{c}^{\infty}(G)$, the invariant integral of $f$ relative to the pair $(G, T)$ is given by

$$
\Phi_{f}^{T}(t)=\Delta_{T}(t) \int_{G / T} f\left({ }^{\dot{x}} t\right) d_{G / T}(\dot{x}), \quad t \in T^{\prime},
$$

where ${ }^{\dot{x}} t=x t x^{-1}, x \in G$, and $d_{G / T}(\dot{x})$ is a suitably normalized invariant measure on $G / T$ (see [3d)] and [5, vol. II, Chapter VIII]).

For fixed $t \in T^{\prime}$, the map $f \mapsto \Phi_{f}^{T}(t), f \in C_{c}^{\infty}(G)$, defines an invariant distribution $\Lambda_{t}$ on $G$. In this paper, we give an explicit formula for the Fourier transform of $\Lambda_{t}$, that is, we determine a linear functional $\hat{\Lambda}_{t}$ such that

$$
\Lambda_{t}(f)=\hat{\Lambda}_{t}(\hat{f}), \quad f \in C_{c}^{\infty}(G) .
$$

In this context, we regard $\hat{f}$ as being defined on the space of invariant eigendistributions on $G$ (see [3b)]). This formula is known in the case $G=S L(2, R)([1],[2],[3 b)])$. A similar inversion formula is given in [4] for the group $S \boldsymbol{L}(2, k), k$ a non-archimedean local field. We note that the proof of the formula in the latter case is quite different from that in the real case due to the form of the characters of the discrete series.

The Plancherel formula for $G$ can be obtained directly from the inversion formula for $\Phi_{f}^{T}(t)$ by applying the differential operator $\Pi^{T}=\partial(\tilde{\omega})$ where $\tilde{\omega}$ denotes the product of the positive roots of the pair $\left(g_{c}, t_{c}\right)$, and then using the fact that there exists a nonzero constant $c$ independent of $f$ such

AMS 1970 subject classifications. Primary 22E30; Secondary 22D10, 43A30.

${ }^{1}$ Supported by NSF GP-28271 and GP-29357. 
that $c f(1)=\Phi_{f}^{T}\left(1 ; \Pi^{T}\right)([3 \mathrm{a})]$, [3c)], [5, vol. II, Chapter VIII]). This proof of the Plancherel formula differs from that of Harish-Chandra [3f)] and enables us to avoid the use of the "principal value integral" ([3e), p. 308], [3f) §15], [5, vol. II, Epilogue]).

The derivation of the inversion formula relies heavily on the work of Harish-Chandra, an account of which may be found in [5]. Throughout this note, we adopt the notation of [5]. Full details will appear elsewhere.

2. The structure of $G$ and $\mathfrak{g}$. As in [3f), §24], we take a subalgebra of $\mathfrak{g}$ isomorphic to $\mathfrak{s l}(2, R)$ with basis elements $H, X, Y$ satisfying

$$
[X, Y]=H, \quad[H, X]=2 X, \quad[H, Y]=-2 Y .
$$

Let $A_{\mathfrak{p}}=\exp \{\boldsymbol{R} H\}$ and set $Z\left(A_{\mathfrak{p}}\right)=K \cap \exp \{\sqrt{ }(-1) \boldsymbol{R} H\}$. Then $Z\left(A_{\mathfrak{p}}\right)$ $=\{1, \gamma\}$, where $\gamma=\exp (\sqrt{ }(-1) \pi H)=\exp (\pi(X-Y)) \neq 1$. We have $A=A_{\mathrm{K}} A_{\mathrm{p}}$, where $A_{\mathrm{K}}=A \cap K$. If $T_{1}$ is the identity component of $A_{\mathrm{K}}$ and $\mathrm{t}_{1}$ is the Lie algebra of $T_{1}$, then $\mathrm{t}=\mathrm{t}_{1}+\mathrm{t}_{2}$ where $\mathrm{t}_{2}=R(X-Y)$, and the Lie algebra of $A$ is $\mathfrak{a}=\mathrm{t}_{1}+R H$. Moreover, if $T_{2}=\exp \left(\mathrm{t}_{2}\right)$, then $T_{2}$ is isomorphic to $S O(2)$ (a circle) and $T_{1} \cap T_{2} \subset Z\left(A_{\mathfrak{p}}\right)$. We have $A_{K}$ $=T_{1} \cup \gamma T_{1}$.

Let $W(G, T)$ and $W(G, A)$ denote the Weyl groups of the pairs $(G, T)$ and $(G, A)$ respectively [3d), §20]. If $w \in W(G, T)$ and $t_{0} \in T^{\prime}$, then we can write $w^{-1} \cdot t_{0}=t_{1}(w) t_{2}(w)$ where $t_{1}(w) \in T_{1}$ and $t_{2}(w) \in T_{2}$ (uniquely up to $\left.T_{1} \cap T_{2}\right)$. If $-\pi<\operatorname{Arg}\left(t_{2}(w)\right)<0\left(0<\operatorname{Arg}\left(t_{2}(w)\right)<\pi\right)$, we shall simply write $-\pi<t_{2}(w)<0\left(0<t_{2}(w)<\pi\right)$.

3. The representations of $G$ and their characters. The unitary character group $\hat{T}$ of $T$ may be identified with a lattice $L_{T}$ in the dual space of $\sqrt{ }(-1)$ t. An element $\tau \in L_{T}$ gives rise to a character in $\hat{T}$ by the formula $\xi_{\tau}(\exp H)=e^{\tau(H)}, H \in \mathrm{t}$. To each $\tau \in L_{T}$ is associated an invariant eigendistribution $\Theta_{\tau}$ on $G$ ([3e), p. 289], [3f), p. 90]). If $\tau$ is regular $(w \cdot \tau \neq \tau$ for every nontrivial $w$ in the Weyl group $\left.W\left(\mathfrak{g}_{c}, \mathrm{t}_{c}\right)\right)$, then $\Theta_{\tau}$ leads directly to the character of a member of the discrete series for $G([3 \mathrm{~g})$, p. 96]). Except for a few special cases, there is no character theoretic interpretation of $\Theta_{\tau}$ for singular $\tau$ presently available.

Let $M$ denote the centralizer of $A_{\mathfrak{p}}$ in $K$. The principal series of unitary representations of $G$ may be indexed by pairs $(\sigma, v)$ where $\sigma$ is an irreducible unitary representation of $M$ and $v \in \hat{A}_{\mathfrak{p}}$. To the representation $\sigma$, one can associate a regular character $\chi\left(=\chi_{\sigma}\right)$ in $\hat{A}_{\mathrm{K}}$. We may then view the principal series as being parameterized by the pairs $(\chi, v)$ in $\hat{A}_{K} \times \hat{A}_{\mathfrak{p}}$ with $\chi$ regular (see [5, vol. II, Epilogue]). For any $\chi \in \hat{A}_{\mathbf{K}}$, we define $\log \chi$ by the equation

$$
\langle\exp H, \chi\rangle=\exp \langle H, \log \chi\rangle, \quad H \in \mathfrak{a}_{K}=\mathfrak{t}_{1} .
$$


There are equivalences among the representations of the discrete series corresponding to the orbits of $W(G, T)$ in the set of regular elements in $L_{T}$. Similarly, there are equivalences among the representations of the principal series corresponding to the orbits of $W(G, A)$ in $\hat{A}_{\boldsymbol{K}} \times \hat{A}_{\mathfrak{p}}$. In the inversion formula, these equivalences are accounted for by the appearance of $W(G, A)$.

4. The inversion formula. Fix $t_{0} \in T^{\prime}$. For any $w \in W(G, T)$, write $w^{-1} \cdot t_{0}=t_{1}(w) t_{2}(w)$ where $t_{1}(w) \in T_{1}$ and $t_{2}(w) \in T_{2}$. (If $T_{1} \cap T_{2}=\{1, \gamma\}$, the decomposition is not unique, but this causes no difficulty.) If $T_{1} \cap T_{2}$ $=\{1\}$, we define $($ see $\$ 2)$

$$
\begin{aligned}
& W^{+}\left(G, T, t_{0}\right)=\left\{w \in W(G, T): 0<t_{2}(w)<\pi\right\}, \\
& W^{-}\left(G, T, t_{0}\right)=\left\{w \in W(G, T):-\pi<t_{2}(w)<0\right\} .
\end{aligned}
$$

For $f \in C_{c}^{\infty}(G)$, set $\Theta_{\tau}(f)=\int_{G} f(x) \Theta_{\tau}(x) d x, \tau \in L_{T}$, and write $T^{(\chi, v)}(f)$ for the character of the principal series associated to the pair $(\chi, v) \in \hat{A}_{\boldsymbol{K}} \times \hat{A}_{\mathfrak{p}}$, $\chi$ regular [5, vol. II, Epilogue]. If $\chi$ is singular, $T^{(\chi, v)}(f)$ can be defined in a natural way.

Let $\Phi_{I}^{+}$be the set of positive imaginary roots of the pair $\left(\mathfrak{g}_{c}, \mathfrak{a}_{c}\right)$ and set

$$
\begin{aligned}
\varepsilon(\chi) & =\operatorname{sgn}\left[\prod_{\alpha \in \Phi_{I}^{+}}(\log \chi, \alpha)\right], & & \chi \text { regular }, \\
& =1, & & \chi \text { singular. }
\end{aligned}
$$

Set $r_{I}=\left[\Phi_{I}^{+}\right]$(we denote the cardinality of a set $J$ by $[J]$ ) and $r=\frac{1}{2}(\operatorname{dim} G-\operatorname{rank} G)$.

TheOREM. Suppose that $f \in C_{c}^{\infty}(G)$ and $t_{0} \in T^{\prime}$. Then, if $T_{1} \cap T_{2}=\{1\}$,

$$
\begin{aligned}
\Phi_{f}^{T}\left(t_{0}\right)= & (-1)^{r} \sum_{\tau \in L_{T}} \overline{\xi_{\tau}\left(t_{0}\right)} \Theta_{\tau}(f) \\
& +(-1)^{r_{1}}(\sqrt{ }(-1) / 2)[W(G, A)]^{-1} \sum_{w \in W^{ \pm}\left(G, T, t_{0}\right)} \operatorname{det}(w) \sum_{\chi \in \hat{A}_{\mathbf{K}}} \varepsilon(\chi) \\
& \times\left[\overline{\chi\left(t_{1}(w)\right)} \int_{-\infty}^{\infty} T^{(\chi, v)}(f) \frac{\sinh \left(v\left(t_{2}(w) \mp \pi\right)\right)}{\sinh (v \pi)} d v\right. \\
& \left.+\overline{\chi\left(\gamma t_{1}(w)\right)} \int_{-\infty}^{\infty} T^{(\chi, v)}(f) \cdot \frac{\sinh \left(v t_{2}(w)\right)}{\sinh (v \pi)} d v\right] .
\end{aligned}
$$

Here, the sums over $W^{+}\left(G, T, t_{0}\right)$ and $W^{-}\left(G, T, t_{0}\right)$ are to be taken separately and the sign in the first integral is taken in the obvious way. If $T_{1} \cap T_{2}=\{1, \gamma\}$, the sums over $W^{+}\left(G, T, t_{0}\right)$ and $W^{-}\left(G, T, t_{0}\right)$ are replaced by a single sum over $W(G, T)$ and the plus sign does not appear in the first integral. 
The principle of the proof is the same as the case of $\operatorname{SL}(2, R)$, that is, the Fourier coefficients of $\Phi_{f}^{T}$ can be expressed in terms of $\Theta_{\tau}(f), \tau \in L_{T}$, along with an integral over the hyperbolic elements in $G$. This latter integral must then be written as an integral over the principal series.

\section{REFERENCES}

1. S. Gelbart, Fourier analysis on matrix space, Mem. Amer. Math. Soc. No. 108 (1971).

2. I. M. Gel'fand, M. I. Graev and I. I. Pjateckiř-Sapiro, Generalized functions. Vol. 6: Theory of representation and automorphic functions, "Nauka", Moscow, 1966; English transl., Saunders, Philadelphia, Pa., 1969. MR 36 \# 3725; 38 \# 2093.

3. Harish-Chandra, a) A formula for semisimple Lie groups, Amer. J. Math. 79 (1957), 733-760. MR 20 \#2633.

b) Harmonic analysis on semisimple Lie groups, Some recent advances in the basic sciences. Vol. 1 (Proc. Annual Sci. Conf., Belfer Grad. School Sci., Yeshiva Univ., New York, 1962-1964), Belfer Graduate School of Science, Yeshiva University, New York, 1966, pp. 35-40. MR 35 \# 1717.

c) Some results on an invariant integral on a semisimple Lie algebra, Ann. of Math. (2) 80 (1964), 551-593. MR 31 \# 4862b.

d) Invariant eigendistributions on a semisimple Lie group, Trans. Amer. Math. Soc. 119 (1965), 457-508. MR 31 \# 4862d.

e) Discrete series for semisimple Lie groups. I, Acta Math. 113 (1965), 241-318. MR 36 \#2744.

f) Two theorems on semisimple Lie groups, Ann. of Math. (2) 83 (1966), 74-128. MR 33 \#2766.

g) Discrete series for semisimple Lie groups. II, Acta Math. 116 (1966), 1-111. MR 36 \#2745.

4. P. J. Sally, Jr. and J. A. Shalika, The Fourier transform on SL(2) over a non-archimedean local field (to appear).

5. G. Warner, Harmonic analysis on semi-simple Lie groups, 2 volumes, Springer-Verlag, Berlin, 1972.

Department of Mathematics, University of Chicago, Chicago, Illinois 60637

Department of Mathematics, University of Washington, Seattle, Washington 98105

Current address: (SAlly) School of Mathematics, Institute for Advanced Study, Princeton, New Jersey 08540 